\title{
The Matter-Antimatter Asymmetry Problem
}

\author{
Brian Albert Robson \\ Department of Theoretical Physics, Research School of Physics and Engineering, The Australian National University, Canberra, \\ Australia \\ Email: brian.robson@anu.edu.au
}

How to cite this paper: Robson, B.A. (2018) The Matter-Antimatter Asymmetry Problem. Journal of High Energy Physics, Gravitation and Cosmology, 4, 166-178. https://doi.org/10.4236/jhepgc.2018.41015

Received: December 21, 2017

Accepted: January 28, 2018

Published: January 31, 2018

Copyright $\odot 2018$ by author and Scientific Research Publishing Inc. This work is licensed under the Creative Commons Attribution International License (CC BY 4.0).

http://creativecommons.org/licenses/by/4.0/

\section{c) (i) Open Access}

\begin{abstract}
The matter-antimatter asymmetry problem, corresponding to the virtual nonexistence of antimatter in the universe, is one of the greatest mysteries of cosmology. According to the prevailing cosmological model, the universe was created in the so-called "Big Bang" from pure energy and it is generally considered that the Big Bang and its aftermath produced equal numbers of particles and antiparticles, although the universe today appears to consist almost entirely of matter rather than antimatter. This constitutes the matter-antimatter asymmetry problem: where have all the antiparticles gone? Within the framework of the Generation Model (GM) of particle physics, it is demonstrated that the asymmetry problem may be understood in terms of the composite leptons and quarks of the GM. It is concluded that there is essentially no matter-antimatter asymmetry in the present universe and that the observed hydrogen-antihydrogen asymmetry may be understood in terms of statistical fluctuations associated with the complex many-body processes involved in the formation of either a hydrogen atom or an antihydrogen atom.
\end{abstract}

\section{Keywords}

Generation Model, Antimatter, Big Bang

\section{Introduction}

The standard model of cosmology [1] assumes that the universe was created in the so-called "Big Bang" from pure energy, and is now composed of about 5\% ordinary matter, $27 \%$ dark matter and $68 \%$ dark energy. It is also generally assumed that the Big Bang produced equal numbers of particles and antiparticles. This leads to the matter-antimatter asymmetry problem since the universe today is considered to consist almost entirely of matter (particles) rather than antimatter (antiparticles): where have all the antiparticles gone? Currently there is no acceptable understanding of this asymmetry problem. 
An understanding of the asymmetry problem requires both knowledge of the physical nature of the Big Bang and a precise definition of matter. Unfortunately, knowledge of the physical nature of the Big Bang is currently far from complete and matter has not been defined precisely within the framework of the Standard Model (SM) [2] of particle physics.

The prevailing model of the Big Bang is based upon the General Theory of Relativity (GTR) [3]. According to this theory, extrapolation of the expansion of the universe backwards in time yields an infinite density and temperature at a finite time, approximately 13.8 billion years ago. Thus the "birth" of the universe appears to be associated with a "singularity". This indicates a breakdown of the GTR but also all the laws of physics. This is a serious impediment to understand the matter-antimatter (i.e. the particle-antiparticle) asymmetry problem. Consequently, this problem will be discussed in terms of the observed nature of the universe, ignoring the singularity.

A consistent definition of the terms matter and antimatter is the following: matter is built of elementary matter particles and antimatter is built of elementary antimatter antiparticles.

In the SM the elementary matter particles are assumed to be the leptons and quarks so that electrons, neutrons and protons, which constitute the $5 \%$ ordinary matter in the universe (92\% hydrogen atoms and $8 \%$ helium atoms [4]), are all matter. In the Generation Model (GM) [5] (see Section 3), the elementary matter particles are "rishons" and the elementary antimatter particles are "antirishons" so that electrons, neutrons and protons are not all matter.

In Section 2 the main attempts, within the framework of the SM, to understand the asymmetry problem will be discussed. In Section 3 we shall introduce the GM of particle physics [5] and summarize the essential differences between the GM and the SM. In Section 4 we shall show how the GM naturally provides an understanding of the asymmetry problem in terms of composite leptons and quarks. Since these composite leptons and quarks are composed of the same kinds of elementary particles and/or antiparticles, their matter and/or antimatter nature is defined in terms of their constituents, contrary to pure convention as in the SM. Section 5 states the conclusions.

\section{Matter-Antimatter Asymmetry Problem and the Standard Model}

Within the framework of the SM the matter-antimatter asymmetry problem is generally considered to be related to the baryon asymmetry problem, i.e. the imbalance of baryonic matter and antibaryonic matter in the observable universe. The universe seems to consist almost entirely of hydrogen and helium atoms rather than antihydrogen and antihelium atoms.

This observation is at first sight rather surprising since the origin of the universe in the Big Bang is generally considered to have produced equal numbers of baryons and antibaryons, which as the universe cooled should have annihilated in pairs back to pure energy, so that the universe would be empty of mat- 
ter. In the SM this is assumed to imply that although the universe was originally perfectly symmetric in baryons and antibaryons, during the cooling period some physical processes contributed to a small imbalance in favor of baryons.

The small excess of baryons over antibaryons indicates that contrary to the current laws of physics, baryon number must be violated in some physical process. This was proposed by Sakharov [6] in 1967. Sakharov proposed a set of three necessary conditions, within the framework of the SM, that a physical process must satisfy to produce baryons and antibaryons at different rates: 1) violation of baryon number; 2) violation of both charge conjugation symmetry, $\mathrm{C}$, and charge conjugation-parity symmetry, $\mathrm{CP}$; and 3) the process must not be in thermal equilibrium. Violation of baryon number is required to produce an excess of baryons over antibaryons, while $\mathrm{C}$ symmetry violation ensures the non-existence of processes, which produce an equivalent excess of antibaryons over baryons. Similarly, violation of CP symmetry is required so that equal numbers of left-handed baryons and right-handed antibaryons as well as equal numbers of right-handed baryons and left-handed antibaryons are not produced. Finally, a departure from thermal equilibrium must play a role so that CPT symmetry does not ensure compensation between processes increasing and decreasing the baryon number [7].

The first Sakharov criterion: violation of baryon number, would be achieved if antiprotons or protons decayed into lighter subatomic particles such as a neutral pion and an electron or positron, respectively. However, there is currently no experimental evidence that such "direct" violations of baryon number occur.

Thus researchers turned their attention to "indirect" violations of baryon number, which are concerned with Sakharov's second criterion: CP violation, which indicates the possibility that some physical processes may distinguish between matter and antimatter.

Both the electromagnetic and strong interactions are symmetric under $\mathrm{C}$ and $\mathrm{P}$, and consequently they are also symmetric under the product $\mathrm{CP}$. However, this is not necessarily the case for the weak interaction, which violates both $\mathrm{C}$ and P symmetries. Indeed, the 1964 discovery [8] of the decay of the long-lived $K^{0}$ meson to two charged pions brought about the surprising conclusion that $\mathrm{CP}$ is also violated in the weak interaction. The violation of $\mathrm{CP}$ in weak interactions implies that such physical processes could lead to indirect violation of baryon number so that matter creation would be preferred over antimatter creation.

In the SM CP violation originates from charge-changing (CC) weak interactions that change the charge and flavor of quarks. The six known quarks consist of three up-like quarks with charge $Q=+\frac{2}{3}$ : up $(u)$, charmed $(c)$ and top $(t)$; and three down-like quarks with charge $Q=-\frac{1}{3}$ : down $(d)$, strange $(s)$ and bottom (b). The CC weak interactions cause each up-like quark to turn into a down-like quark and vice-versa. The transition amplitudes for the nine combinations are 
given by the Cabibbo-Kobayashi-Maskawa (CKM) matrix elements [9] [10].

Prior to the quark model of Gell-Mann [11] and Zweig [12], Cabibbo [9] in 1963 had introduced some of the matrix elements of the CKM matrix in order to preserve the universality of the CC weak interaction. In 1973 Kobayashi and Maskawa [10] discovered that for the case of three generations of quarks the CC weak interactions may violate $\mathrm{CP}$. However, the Kobayashi-Maskawa CP violation was found to be tiny, primarily because of the smallness of the relevant matrix elements. Consequently, any physical process that produces more matter than antimatter would have been ineffectual. Although the excess of matter over antimatter is generally considered to have been only one part in a billion, the effect of the Kobayashi-Maskawa CP violation process falls far short of even this very small amount by many orders of magnitude. Indeed it is estimated that the baryon excess produced by the Kobayashi-Maskawa $\mathrm{CP}$ violation process is only sufficient to provide the baryons of a single galaxy in the universe, which comprises billions of galaxies.

The third criterion of Sakharov: departure from thermal equilibrium, is generally assumed to also occur within the electroweak sector of the SM during the so-called electroweak phase transition [13]. This is assumed to be a first order transition between the state in which the $W$ and $Z$ gauge bosons are massless to a state in which they are massive. The massive $W$ and $Z$ bosons are assumed to arise as a result of some unknown mechanism which breaks the electroweak symmetry, and it is during this electroweak symmetry breaking that departure of thermal equilibrium takes place.

Thus the SM does provide possible physical processes, which satisfy all three necessary criteria of Sakharov. However, the assumed physical processes do not seem capable of providing an acceptable explanation for the matter-antimatter asymmetry. The general conclusion is that physics beyond the SM is required for this purpose.

\section{The Generation Model}

The Generation Model (GM) has been developed over many years [14] [15] [16] [17] [18] and is an alternative model to the SM of particle physics. The current version of the GM is described in detail in Reference [5]. There are three essential differences between this GM and the SM: 1) the classification of the leptons and quarks in terms of additive quantum numbers; 2) the roles played by the mass eigenstate quarks and the weak eigenstate quarks; and 3) the nature of the weak interactions. These are discussed in detail in Reference [19]. For the purpose of the present paper, only the essential differences 1) and 2) will be discussed more briefly below.

The GM employs a unified classification scheme for the leptons and quarks rather than the non-unified scheme of the SM. This unified system is based upon the use of only three additive quantum numbers rather than the nine independent additive quantum numbers of the SM. These three additive quantum numbers 
are charge $(Q)$, particle number $(p)$ and generation quantum number $(g)$. The non-unified system of the SM uses four additive quantum numbers for leptons: charge $(Q)$, lepton number $(L)$, muon lepton number $\left(L_{\mu}\right)$ and tau lepton number $\left(L_{\tau}\right)$ plus six additive quantum numbers for quarks: charge $(Q)$, baryon number $(A)$, strangeness $(S)$, charm $(C)$, bottomness $(B)$ and topness $(T)$.

Comparison of the two models, SM and GM, indicates that they have only one additive quantum number in common, namely electric charge $Q$. The second additive quantum number of the GM, particle number $p$ replaces both lepton number $L$ and baryon number $A$ of the SM. The third additive quantum number of the $\mathrm{GM}$, generation quantum number $g$ effectively replaces the remaining six additive quantum numbers of the SM. Thus the GM provides both a simpler and unified classification scheme for leptons and quarks. Indeed the GM classification scheme indicates that leptons and quarks are intimately related and led to composite models of leptons and quarks, which in turn led to new paradigms for both gravity and mass [18] [20].

An important feature of the GM classification scheme is that all three additive quantum numbers, $Q, p$ and $g$, are required to be conserved in all leptonic and hadronic processes. In particular, the generation quantum number $g$ is strictly conserved in weak interactions unlike some of the quantum numbers of the SM, e.g. strangeness $S$. This latter requirement led to a new treatment of quark mixing in hadronic processes [17]: the GM differs from the SM in two fundamental ways that are essential to preserve the universality of the CC weak interaction for hadronic processes.

First, the GM postulates that the mass eigenstate quarks of the same generation, e.g. $(u, d)$, form weak isospin doublets and couple with the full strength of the CC weak interaction, like the lepton doublets. Unlike the SM, the GM requires that there is no coupling between mass eigenstate quarks from different generations. This latter requirement corresponds to the conservation of the generation quantum number $g$ in the CC weak interaction processes.

Second, the GM postulates that hadrons are composed of weak eigenstate quarks, rather than the corresponding mass eigenstate quarks as in the SM. Thus the GM differs from the SM in that it treats quark mixing differently from the method introduced by Cabibbo [9] and employed in the SM. Essentially, in the GM, the quark mixing is placed in the quark states (wave functions) rather than in the CC weak interactions. Thus in the GM, the proton is considered to consist of two up quarks and one mixed $\left(d^{\prime}\right)$ quark, which is a linear superposition of the down quark, the strange quark and the bottom quark:

$$
d^{\prime}=V_{u d} d+V_{u s} s+V_{u b} b,
$$

where $V_{i j}$ are the elements of the CKM matrix [9] [10], rather than two up quarks and one down quarks as in the SM. This allows a unified and simpler classification of both leptons and quarks in terms of only three additive quantum numbers, $Q, p$ and $g$, each of which is conserved in all interactions. It should be noted that in both the GM and the SM, the weak eigenstate up quark is assumed to be identical 
with the mass eigenstate up quark, i.e. there is no mixing with the other up-like quarks, $(c)$ and $(t)$, unlike the down-like quarks.

The development of the unified GM classification scheme for leptons and quarks indicated that leptons and quarks are intimately related and led to the development of composite versions of the GM. It should be noted that this is not possible in terms of the non-unified classification scheme of the SM, involving different additive quantum numbers for leptons than for quarks and the non-conservation of some additive quantum numbers, such as strangeness, in the case of quarks. As indicated earlier, this intimate relationship between leptons and quarks as a consequence of the unified classification scheme leads to a unified description of the matter and/or antimatter nature in terms of their particle number $p$.

Another essential difference between the GM and the SM is that in the GM, the leptons and quarks are composite particles rather than elementary particles as in the SM. In the GM, both leptons and quarks have a substructure, consisting of spin-1/2 massless particles, rishons and/or antirishons, each of which carries a single color charge. The term "rishon" from the Hebrew word for "first" or "primary" was introduced by Harari [21] in 1979. The constituents of leptons and quarks are bound together by strong color interactions, mediated by massless vector hypergluons, acting between the color charged rishons and/or antirishons. These strong color interactions of the GM are analogous to the strong color interactions of the SM, mediated by massless vector gluons, acting between color charged elementary quarks and/or antiquarks. In the GM, the strong color interaction has been taken down one layer of complexity to describe the composite nature of leptons and quarks. The only essential difference between the strong color interactions of the GM and the SM is that the former acts between color charged rishons and/or antirishons, while the latter acts between color charged elementary quark and/or antiquarks. For historical reasons we use the term "hypergluons" for the mediators of the strong color interactions at the rishon level, rather than the term "gluons" as employed in the SM, although the effective color interaction between composite quarks and/or composite antiquarks is very similar to that between the elementary quarks and/or elementary antiquarks of the SM. The substructure of leptons and quarks is described in detail in Reference [5].

In the GM the elementary rishons and antirishons are required to have the same three kinds of additive quantum numbers as the composite leptons and quarks. Table 1 gives the three additive quantum numbers allotted to the three kinds of rishons of the GM, $T, V$ and $U$. For each rishon additive quantum number $N$, the corresponding antirishon has the additive quantum number $-N$. In particular, each rishon has $p=+\frac{1}{3}$, while each antirishon has $p=-\frac{1}{3}$. Thus the particle number $p$ allotted to a composite lepton or quark reflects its degree of particle or antiparticle nature. In the GM the quarks are composed of both rishons and antirishons so that they have both a matter and an antimatter nature. 
Table 1. GM additive quantum numbers for rishons.

\begin{tabular}{cccc}
\hline Rishon & $Q$ & $p$ & $g$ \\
\hline$T$ & $+\frac{1}{3}$ & $+\frac{1}{3}$ & 0 \\
$V$ & 0 & $+\frac{1}{3}$ & 0 \\
$U$ & 0 & $+\frac{1}{3}$ & -1 \\
\hline
\end{tabular}

Table 2 displays both the structures and their additive quantum numbers of the first generation of composite leptons and quarks in the GM. The structures of the first generation are based upon the structures of the schematic models of Harari [21] and Shupe [22] with the $\bar{V}$-antirishon replacing the $V$-rishon of the schematic models.

Each lepton of the first generation is assumed to be colorless, consisting of three rishons (or antirishons), each with a different color (or anticolor), analogous to the baryons (or antibaryons) of the SM. The leptons are built out of $T$-rishons and $V$-rishons or their antiparticles $\bar{T}$ and $\bar{V}$, all of which have generation quantum number $g=0$.

In the GM it is assumed that each quark of the first generation is a composite of a colored rishon and a colorless rishon-antirishon pair, $(T \bar{V})$ or $(\overline{T V})$, so that the quarks carry a color charge. Similarly, the antiquarks are a composite of an anticolored antirishon and a colorless rishon-antirishon pair, so that the antiquarks carry an anticolor charge.

The rishon structures of the second generation particles are assumed to be the same as the corresponding particles of the first generation plus the addition of a colorless rishon-antirishon pair, $\Pi$, where

$$
\Pi=[(\bar{U} V)+(\bar{V} U)] / \sqrt{2},
$$

which is a quantum mechanical mixture of $(\overline{U V})$ and $(\bar{V} U)$, which have $Q=p=0$ but $g= \pm 1$, respectively. In this way, the pattern for the first generation is repeated for the second generation. Equation (2) indicates that the generation quantum number $g$ for each second generation particle has two possible values, \pm 1 , although in any given transition the generation quantum is required to be conserved.

Similarly, the rishon structures of the third generation particles are assumed to be the same as the corresponding particles of the first generation plus the addition of two $\Pi$ rishon-antirishon pairs so that the pattern of the first and second generation is continued for the third generation. The structure

$$
\Pi \Pi=[(\bar{U} V)(\bar{U} V)+(\bar{U} V)(\bar{V} U)+(\bar{V} U)(\bar{U} V)+(\bar{V} U)(\bar{V} U)] / 2
$$

indicates that the generation quantum number for each third generation particle has three possible values $g=0, \pm 2$, although in any given transition the 
Table 2. GM of first generation of leptons and quarks.

\begin{tabular}{ccccc}
\hline Particle & Structure & $Q$ & $p$ & $g$ \\
\hline$e^{+}$ & $T T T$ & +1 & +1 & 0 \\
$\bar{d}$ & $T T \bar{V}$ & $+\frac{2}{3}$ & $+\frac{1}{3}$ & 0 \\
$v_{e}$ & $T \bar{V} \bar{V}$ & $+\frac{1}{3}$ & $-\frac{1}{3}$ & 0 \\
$\bar{v}_{e}$ & $\bar{V} \bar{V}$ & 0 & -1 & 0 \\
$d$ & $V V V$ & 0 & +1 & 0 \\
$\bar{u}$ & $\overline{T V V}$ & $-\frac{1}{3}$ & $+\frac{1}{3}$ & 0 \\
$e^{-}$ & $\overline{T T V}$ & $-\frac{2}{3}$ & $-\frac{1}{3}$ & 0 \\
\hline
\end{tabular}

generation quantum number is required to be conserved.

Equations (2) and (3) indicate that the weak eigenstate quark $d^{\prime}$ has charge $Q=-\frac{1}{3}$ and particle number $p=+\frac{1}{3}$ although each component has different values of the generation quantum number $g$.

\section{Matter-Antimatter Asymmetry Problem and the Generation Model}

In Section 2 we have discussed the matter-antimatter asymmetry problem within the framework of the SM. For many decades now the SM has been unable to provide an understanding of the asymmetry problem. The main reason is that the SM assumes that the leptons and quarks are all elementary particles so that the matter/antimatter nature of leptons and quarks may be decided by pure convention. In the SM both leptons and quarks are assumed to be matter particles.

In the SM it is assumed that the Big Bang initially produces numerous elementary particle-antiparticle pairs such as electron-positron pairs and quark-antiquark pairs by converting energy into mass according to $m=E / c^{2}$. Thus the early universe consisted of a soup of particle-antiparticle pairs continually being created and annihilated. Later, as the universe cooled, the quarks and antiquarks would form protons, neutrons, antiprotons, antineutrons, etc., and eventually, together with electrons and positrons, atoms of hydrogen, antihydrogen, helium and antihelium. These would later annihilate in pairs until only atoms of hydrogen and helium prevailed.

However, it is unlikely that either electrons or positrons would prevail so that neither hydrogen atoms nor antihydrogen atoms would prevail, since both electrons and positrons are elementary particles in the SM and consequently the creation and annihilation of electron-positron pairs constitute a unique process.

In the GM it is expected that the Big Bang initially produces numerous ele- 
mentary rishon-antirishon pairs corresponding to the three kinds of rishons. Then, as the universe cooled, the rishons and antirishons would form leptons, quarks and their antiparticles and eventually atoms of hydrogen, antihydrogen, helium and antihelium. Later these would annihilate in pairs until only atoms of hydrogen and helium prevailed.

In order to understand the matter-antimatter asymmetry in the GM, it is necessary to define the matter/antimatter nature of composite particles. Historically, the term "particle" defines matter that is naturally occurring, i.e. electrons, protons, hydrogen atoms, etc. This is consistent within the SM in which the electron and the up and down quarks are elementary particles and are defined conventionally as matter. However, it is inconsistent within the GM in which the electron and the up and down quarks are composite particles consisting of rishons and/or antirishons. In the GM, rishons are considered to be matter while antirishons are considered to be antimatter, since rishon-antirishon pairs are considered to be created/annihilated in the Big Bang.

In the GM (see Table 1), the elementary rishons and antirishons have $p=+\frac{1}{3}$ and $p=-\frac{1}{3}$, respectively. Thus the particle number $p$ allotted to a composite lepton or quark reflects its degree of matter or antimatter nature. In the GM the quarks are composed of both rishons and antirishons so that they have both a matter and an antimatter nature. On the other hand an electron, consisting of three $\bar{T}$-antirishons, has $p=-1$ and is pure antimatter.

The solution of the matter-antimatter asymmetry problem involves the particle number additive quantum number $p$ of the GM: in particular the values of $p$ corresponding to a weak eigenstate $u$-quark $\left(p=+\frac{1}{3}\right)$, a weak eigenstate $d^{\prime}$-quark $\left(p=+\frac{1}{3}\right)$ and an electron $(p=-1)$. The values of $p=+\frac{1}{3}$ of the weak eigenstate quarks correspond to the values of their baryon number in the $\mathrm{SM}$, while the value of $p=-1$ of the electron, corresponds to minus the value of the lepton number of the electron in the SM. In the GM, the electron consists entirely of antirishons, i.e. antiparticles, while in the SM it is assumed to be a particle, although as we have indicated earlier, there is no a priori reason for this assumption based solely upon convention. It should be noted that the matter/antimatter nature of an electron in the GM is not merely a revised definition of the term "matter" but is a requirement for consistency of the nature of the constituents of the electron and the initial particle-antiparticle nature of the universe in the Big Bang: the elementary particles in the SM, leptons and quarks, and in the GM, rishons, are different.

In the GM the proton consists of three weak eigenstate quarks, two $u$-quarks and one $d^{\prime}$-quark, so that the proton has particle number $p=+1$. Consequently, a hydrogen atom, consisting of one proton and one electron has particle number $p=0$. The hydrogen atom in the GM consists of an equal number of rishons and antirishons, so that $p=0$ and there is no asymmetry of matter and anti- 
matter there.

In the GM the neutron is composed of three weak eigenstate quarks, one $u$-quark and two $d^{\prime}$-quarks, so that the neutron also has particle number $p=+1$. Consequently, a helium atom, consisting of two protons, two neutrons and two electrons has particle number $p=+2$ : the helium atom in the GM consists of six more rishons than antirishons, i.e. more matter than antimatter. In the GM, during the formation of helium in the aftermath of the Big Bang, it is assumed that an equivalent surplus of antimatter was formed as neutrinos, which have $p=-1$, so that overall equal numbers of rishons and antirishons prevailed. This assumption is a consequence of the conservation of $p$ in all interactions in the GM.

A further consequence of the above assumption concerning the conservation of $p$ in all interactions is that if the initial state of the universe has $p=0$ following the production of equal numbers of particles and antiparticles in the Big Bang then the GM predicts that the present state of the universe should also have $p=0$.

To summarize: the ordinary matter present in the universe has an overall particle number of $p=0$, so that it contains equal numbers of both rishons and antirishons. This implies that the original antimatter created in the Big Bang is now contained within the stable composite leptons, i.e. electrons and neutrinos, and the stable composite quarks, i.e. the weak eigenstate $u$-quarks and $d^{\prime}$-quarks, comprising the protons and neutrons. The hydrogen, helium and heavier atoms, following the fusion processes in stars, all consist of electrons, protons and neutrons. This explains where all the antiparticles have gone. Thus there is no matter-antimatter asymmetry in the present universe. However, it does not explain why the universe consists primarily of hydrogen atoms and not antihydrogen atoms. It is suggested that this hydrogen-antihydrogen asymmetry may be understood as follows.

In the GM, an antihydrogen atom consists of the same six rishons and six antirishons as a hydrogen atom, although the rishons and antirishons are differently arranged in the two systems. This implies that both hydrogen atoms and antihydrogen atoms should be formed during the aftermath of the Big Bang with about the same probability. In fact, estimates from the cosmic microwave background data [23] suggest that for every billion hydrogen-antihydrogen pairs there was just one extra hydrogen atom. It is proposed that this extremely small difference, one extra hydrogen atom in $10^{9}$ hydrogen-antihydrogen pairs, may arise from statistical fluctuations associated with the complex many-body processes involved in the formation of either a hydrogen atom or an antihydrogen atom. The uniformity of the universe [23], in particular, the lack of antihydrogen throughout the universe, indicates that the above statistical fluctuations took place prior to the "inflationary period" [24] [25] associated with the Big Bang scenario.

\section{Conclusions and Discussion}

Within the framework of the GM of particle physics, it has been demonstrated 
that the matter-antimatter asymmetry problem may be understood in terms of the particle additive quantum number $(p)$ and the composite nature of the leptons and quarks of the GM. The ordinary matter presented in the universe has an overall particle number $p=0$, so that it contains the same number of particles (rishons) as antiparticles (antirishons).

This implies that the original antimatter created in the Big Bang is now contained within the stable composite leptons, the electrons and neutrinos, and the stable composite quarks, the weak eigenstate up and down quarks that comprise the protons and neutrons, within the hydrogen, helium and heavier atoms of the universe.

Thus there is no matter-antimatter asymmetry in the present universe. However, there does exist a hydrogen-antihydrogen asymmetry: the present universe consists predominantly of hydrogen atoms and virtually no antihydrogen atoms. In the SM this is tantamount to the matter-antimatter asymmetry, since both protons and electrons are assumed to be matter. In the GM this is not the case, since both hydrogen and antihydrogen atoms contain the same number of rishons as the number of antirishons.

Thus there are two main conclusions: 1) there is no matter-antimatter asymmetry in the present universe; and 2) it is suggested that the observed extremely small hydrogen-antihydrogen asymmetry (one additional hydrogen atom in a billion hydrogen-antihydrogen pairs) may be understood in terms of statistical fluctuations associated with the complex many-body processes involved in the formation of either a hydrogen atom or an antihydrogen atom.

Finally it should be noted that if the Big Bang produced equal numbers of particles and antiparticles so that the initial state of the universe had particle number $p=0$, i.e. no matter-antimatter asymmetry, then the GM predicts that the present state of the universe should also have $p=0$, since particle number $p$ is conserved in all interactions.

\section{Acknowledgements}

I am grateful to N. H. Fletcher, D. Robson and L. J. Tassie for helpful discussions.

\section{References}

[1] Ade, P.A.R., et al. (Planck Collaboration) (2014) Planck 2013 Results. I. Overview of Products and Scientific Results. Astronomy and Astrophysics, 571, Article ID: A1.

[2] Gottfried, K. and Weisskopf, V.F. (1984) Concepts of Particle Physics. Vol. 1. Oxford University Press, New York.

[3] Einstein, A. (1916) The Basics of General Relativity Theory. Annalen der Physik, 49, 769-822. https://doi.org/10.1002/andp.19163540702

[4] Morris, R. (1993) Cosmic Questions. Wiley, New York.

[5] Robson, B.A. (2012) The Generation Model of Particle Physics. In: Kennedy, E., Ed., Particle Physics, InTech, Rijeka, 1-28. https://doi.org/10.5772/35071

[6] Sakharov, A.D. (1967) Violation of CP Invariance C Asymmetry and Baryon Asym- 
metry of Universe. JETP Letters, 5, 24-27.

[7] Farrar, G.R. and Shaposhnikov, M.E. (1993) Baryon Asymmetry of the Universe in the Minimal Standard Model. Physical Review Letters, 70, 2833-2836.

https://doi.org/10.1103/PhysRevLett.70.2833

[8] Christenson, J.H., Cronin, J.W., Fitch, V.I. and Turlay, R. (1964) Evidence for the $2 \pi$ Decay of the $K_{2}^{0}$ Meson. Physical Review Letters, 13, 138-140. https://doi.org/10.1103/PhysRevLett.13.138

[9] Cabibbo, N. (1963) Unitary Symmetry and Leptonic Decays. Physical Review Letters, 10, 531-533. https://doi.org/10.1103/PhysRevLett.10.531

[10] Kobayashi, M. and Maskawa, T. (1973) CP-Violation in Renormalizable Theory of Weak Interaction. Progress of Theoretical Physics, 49, 652-657. https://doi.org/10.1143/PTP.49.652

[11] Gell-Mann, M. (1964) A Schematic Model of Baryons and Mesons. Physics Letters, 8, 214-215. https://doi.org/10.1016/S0031-9163(64)92001-3

[12] Zweig, G. (1964) An SU 3 Model for Strong Interaction Symmetry and Its Breaking. CERN-TH 412, 80 p.

[13] Dine, M. and Kusenko, A. (2004) Origin of the Matter-Antimatter Asymmetry. Reviews of Modern Physics, 76, 1-30. https://doi.org/10.1103/RevModPhys.76.1

[14] Robson, B.A. (2002) A Generation Model of the Fundamental Particles. International Journal of Modern Physics E, 11, 555-566.

https://doi.org/10.1142/S0218301302001125

[15] Robson, B.A. (2004) Relation between Strong and Weak Isospin. International Journal of Modern Physics E, 13, 999-1018. https://doi.org/10.1142/S0218301304002521

[16] Robson, B.A. (2005) A Generation Model of Composite Leptons and Quarks. International Journal of Modern Physics E, 14, 1151-1169. https://doi.org/10.1142/S0218301305003776

[17] Evans, P.W. and Robson, B.A. (2006) Comparison of Quark Mixing in the Standard and Generation Models. International Journal of Modern Physics E, 15, 617-625. https://doi.org/10.1142/S0218301306004077

[18] Robson, B.A. (2011) A Quantum Theory of Gravity Based on a Composite Model of Leptons and Quarks. International Journal of Modern Physics E, 20, 733-745. https://doi.org/10.1142/S0218301311018198

[19] Robson, B.A. (2013) Progressing beyond the Standard Model. Advances in High Energy Physics, 2013, Article ID: 341738.

[20] Robson, B.A. (2009) The Generation Model and the Origin of Mass. International Journal of Modern Physics E, 18, 1773-1780. https://doi.org/10.1142/S0218301309013786

[21] Harari, H. (1979) A Schematic Model of Quarks and Leptons. Physics Letters B, 86, 83-86. https://doi.org/10.1016/0370-2693(79)90626-9

[22] Shupe, M.A. (1979) A Composite Model of Leptons and Quarks. Physics Letters B, 86, 87-92. https://doi.org/10.1016/0370-2693(79)90627-0

[23] Lincoln, D. (2012) Understanding the Universe from Quarks to the Cosmos. World Scientific, Singapore. https://doi.org/10.1142/8313

[24] Guth, A.H. (1981) Inflationary Universe-A Possible Solution to the Horizon and Flatness Problems. Physical Review D, 23, 347-356.

https://doi.org/10.1103/PhysRevD.23.347 
[25] Linde, A.D. (1982) A New Inflationary Universe Scenario-A Possible Solution of the Horizon, Flatness, Homogeneity, Isotropy and Primordial Monopole Problems. Physics Letters B, 108, 389-393. https://doi.org/10.1016/0370-2693(82)91219-9 